\title{
Local and Global Stability of Symmetric Heterogeneously-Delayed Control Systems
}

\author{
Yueping Zhang and Dmitri Loguinov* \\ Texas A\&M University, College Station, TX 77843 \\ Email: \{yueping, dmitri\}@cs.tamu.edu
}

\begin{abstract}
Stability proofs of nonlinear congestion control systems under heterogeneous feedback delays are usually difficult and involve a fair amount of effort. In this paper, we show that there exist a class of congestion control methods that admit very simple proofs of asymptotic stability and allow control equations to be delay-independent. This is in contrast to most previous work, which requires that each flow (and sometimes each router) adapt its control-loop constants based on the feedback delay and/or the length of the corresponding end-to-end path. Our new congestion control method, which we call Max-min Kelly Control (MKC), builds upon Kelly's original work in [4] and allows end-flows to be stable and fair regardless of network feedback delays or the number of hops in their end-to-end paths. Using basic matrix algebra and discrete control theory, we show MKC's local asymptotic stability under heterogeneous, directional feedback delays. We also offer a simple proof of its global asymptotic stability assuming constant feedback delay.
\end{abstract}

\section{INTRODUCTION}

Recent research efforts [2], [3], [4], [5], [6], [7], [8], [9], [10], [13], [14] offer an innovative interpretation of Internet congestion control mechanisms from the perspective of economics. One representative method in this category is the framework originated by Kelly et al. [4], in which both optimization and game theory are used to model the network and the end-flows. Kelly's approach models end-users as distributed and non-cooperative entities, where each entity implements an independent strategy to maximize its locally maintained utility and minimize the prices paid for using network resources. Stimulated by Kelly's work, subsequent studies [2], [10], [11], [13], [14] and various extensions [5], [6], [7], [8], [9] of Kelly controls have formed a distinct research area inside current Internet congestion control.

Most current studies [4], [5], [7], [8], [9], [12], [13], [14] of Kelly controls are conducted on the basis of a continuoustime fluid model; however, all real networks are discrete and thus may exhibit different stability conditions from those derived using continuous fluid models. Moreover, local stability conditions derived in prior work [2], [10], [13], [14] require that parameters of the control equation be adaptively tuned according to feedback delays $D_{i}$, which is undesirable in practice since it leads to unfairness between the flows and oscillations when the delays are not properly estimated by the users. Finally, prior work typically assumes that $D_{i}(t)=$

*Supported by NSF grants CCR-0306246, ANI-0312461, and CNS0434940
$D_{i}$ is constant over time, which leads to uncertainty as to whether (and how) their stability conditions hold under random (stochastic) delay $D_{i}(t)$ often found in the real Internet.

To overcome these limitations, this paper provides a new insight into discrete Kelly controls and demonstrates how to stabilize them under random (heterogeneous) feedback delays and constant gain parameters of the control equation. We accomplish this task through a simple modification to the control loop of Kelly's controller and offer a fresh look at this framework by associating it with max-min fairness instead of the original proportional fairness [4]. Accordingly, we call this new controller Max-min Kelly Control $\left(\mathrm{M}_{\mathrm{C}}\right)$ and demonstrate that it is both locally asymptotically stable regardless of feedback delays (which can be random or otherwise) and globally asymptotically stable under constant delay $D$.

The rest of this paper is organized as follows. In section II, we construct the system model and clarify the assumptions used throughout the paper. In section III, we discuss the details of $\mathrm{MKC}$. In section IV, we present several generic results on stability of delayed systems and prove local asymptotic stability of $\mathrm{MKC}^{\mathrm{K}}$ under heterogeneous delay. In section $\mathrm{V}$, we examine $\mathrm{MK} C$ 's global asymptotic stability under constant feedback delay. In section VI, we conclude our work and suggest directions for future research.

\section{Modeling Assumptions And Previous Work}

\section{A. Delayed Congestion Control Systems}

Assume that a network system consists of $N$ users, $M$ resources, and a certain number of data links that connect all these components. Each user $i$ is identified by a route $r_{i}$. All network resources (routers) continuously send congestion feedback to those users in whose path they appear (typically, this information is inserted into all passing packets and then relayed back to the source in positive acknowledgments). Using this feedback information, each source updates its sending rate according to some control equation with the goal to maintain a fair and oscillation-free sharing of network resources.

We next describe how directional and heterogeneous feedback delays are introduced in the control loop. Delays in network feedback arise from both the transmission/propagation time along the data links and the queuing delays at each of the intermediate routers. Consider an 


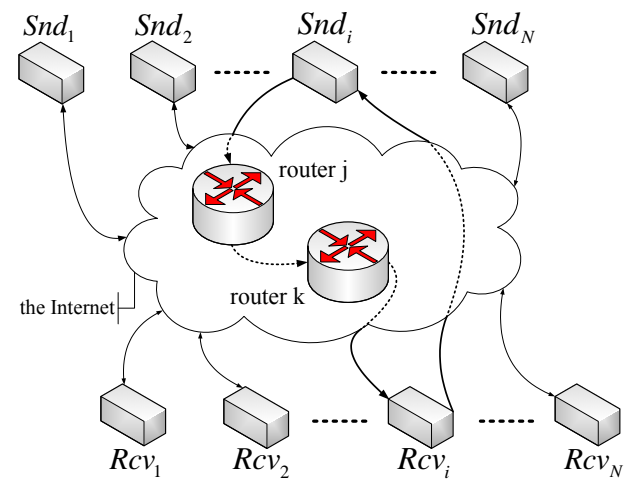

Fig. 1. Model of the network and directional feedback delays.

illustration in Figure 1, where routers $j$ and $k$ are on the path of sender (user) $i$. The time lag for a packet to travel from sender $i$ to router $j$ is denoted by forward delay $D_{i j}$, while the delay from router $j$ to the receiver and subsequently from the receiver back to the sender is denoted by backward

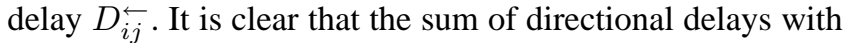
respect to each router is the round trip delay of user $i$, i.e., $D_{i}=D_{i j} \overrightarrow{i j}+D_{i j}^{\leftarrow}=D_{i k}+D_{i k}^{\leftarrow}$.

Under this framework, we next review the class of utility-based controllers proposed in [4] and investigate their delayed stability in the rest of this paper.

\section{B. Related Work}

Recall that the classic Kelly control implements the primal algorithm of the network optimization problem described in [4]. In contrast to the significant research effort [4], [5], [7], [8], [9], [12], [13], [14] put into the continuoustime analysis of Kelly control, Johari et al. introduced a discrete-time version of Kelly's control equation [2]:

$x_{i}(n)=x_{i}(n-1)+\kappa_{i}\left(\omega_{i}-x\left(n-D_{i}\right) \sum_{j \in r_{i}} \mu_{j}\left(n-D_{i j}^{\leftarrow}\right)\right)$,

where $\kappa_{i}$ is a strictly positive gain parameter and $\omega_{i}$ is interpreted as the willingness of user $i$ to pay the price for using the network. According to this notation, $D_{i}=1$ means instantaneous (i.e., most-recent) feedback and $D_{i} \geq$ 2 means delayed feedback. In $(1), \mu_{j}(n)$ is the congestion indication function of resource $j$, which is given by:

$$
\mu_{j}(n)=p_{j}\left(\sum_{u: j \in r_{u}} x_{u}\left(n-D_{u j}^{\vec{j}}\right)\right),
$$

where $p_{j}(\cdot)$ is the price charged by resource $j$. Notice that $p_{j}$ depends only on the combined rate of all flows passing through router $j$ at time $n$.

Next, recall that for a fixed feedback delay $D$, system (1)-(2) is locally asymptotically stable if [2]:

$$
\kappa_{i} \sum_{j \in r_{i}}\left(\left.\left(p_{j}+p_{j}^{\prime} \sum_{u: j \in r_{u}} x_{u}\right)\right|_{x_{u}^{*}}\right)<2 \sin \left(\frac{\pi}{2(2 D-1)}\right),
$$

where $x_{u}^{*}$ is the stationary point of user $u$ and $p_{j}(\cdot)$ is assumed to be differentiable at $x_{u}^{*}$. Additionally, Massoulié and Vinnicombe investigate Kelly's framework under heterogeneous feedback delays based on a continuous-time fluid model and derive sufficient stability conditions, which refine the upper bound in (3) to $1 / D_{i}[10]$ and $\pi /\left(2 D_{i}\right)$ [13], respectively. However, the analysis of discrete stability under heterogeneous delays or the analytical understanding of global stability are missing from the current picture.

Note that all recent studies and proposed controllers [2], [10], [13], [14] based on Kelly's framework require that end-users adapt their parameter $\kappa_{i}$ inverse proportionally to $D_{i}$ (observe in (3) that the right-hand side tends to zero for large $D$ ). Forcing the end-flows to keep $\kappa_{i} \sim 1 / D_{i}$ leads to problems since users are often not aware of the increase in their delay until after oscillations have started and are not able in practice to properly adjust their gain parameter $\kappa_{i}$ in response to such increases in $D_{i}$. Even if we assume that each user can track delay $D_{i}$ and keep $\kappa_{i}$ normalized by $D_{i}$, the resulting system becomes unfair and favors users with smaller RTTs.

The second problem with Kelly's framework is that its stability condition depends on the number of routers along the end-to-end path $r_{i}$ (observe in (3) that the summation term can be arbitrarily high when the number of resources in set $r_{i}$ is high). This leads to another practical issue since each user must now keep $\kappa_{i}$ inversely proportional to the length of the path $r_{i}$, i.e., $\kappa_{i} \sim 1 /\left|r_{i}\right|$. Besides the fact that many flows may not know their exact value of $\left|r_{i}\right|$, there is also the issue of unfairness since not everyone agrees that users with shorter paths should get more throughout.

We should also note that Ying et al. [15] recently established delay-independent stability conditions for a family of utility functions and a generalized controller (1). Their work is similar in spirit to ours; however, the analysis and proposed methods are different.

\section{MAX-Min Kelly CONTROL (MKC)}

To improve the practical aspects of discrete Kelly controls and decouple the delay and path length from gain parameters, in [16], we proposed a new discrete-time congestion control method based on several modifications to the classic Kelly control (1)-(2).

Our first change involves proper selection of the reference rate in (1), which currently applies feedback information about rate $x\left(n-D_{i}\right)$ to the most-recent rate $x(n-1)$. Our second improvement removes the dependency of stability on the number of resources along path $r_{i}$, which we accomplish by feeding back the packet loss from the most congested router in $r_{i}$. Thus, the end-user equation becomes:

$$
x_{i}(n)=x_{i}\left(n-D_{i}\right)+\alpha-\beta \eta_{i}(n) x_{i}\left(n-D_{i}\right),
$$

where parameters $\alpha=\kappa_{i} \omega_{i}, \beta=\kappa_{i}$ are fixed for all users and $\eta_{i}(n)$ is the congestion indication function of user $i$ :

$$
\eta_{i}(n)=\max _{j \in r_{i}} p_{j}\left(n-D_{i j}^{\leftarrow}\right)
$$


Here, $p_{j}(\cdot)$ is the packet loss of router $j$ and depends on the aggregate input rate:

$$
p_{j}(n)=p_{j}\left(\sum_{u \in s_{j}} x_{u}\left(n-D_{u j}^{\vec{j}}\right)\right),
$$

where $s_{j}$ is the set of users passing through router $j$. We call this new controller (4)-(6) Max-min Kelly Control (MKC) [16].

In particular, we can also specify $p_{j}(n)$ with the following standard packet loss function:

$$
p_{j}(n)=\frac{\sum_{u \in s_{j}} x_{u}\left(n-D_{u j}^{\vec{u}}\right)-C_{j}}{\sum_{u \in s_{j}} x_{u}\left(n-D_{u j}^{\vec{j}}\right)},
$$

where $C_{j}$ is the capacity of router $j$. Besides proving max-min fairness, (7) also allows "negative" packet-loss feedback when the bottleneck resource is under-utilized (i.e., the combined rate of all flows passing through the resource is less than its capacity). As we show later in the paper, this change improves the convergence rate to link utilization from linear to exponential. Hence, the resulting controller is called Exponential $\mathrm{MKC}\left(\mathrm{EMKC}_{\mathrm{C}}\right)$ [16].

In what follows in this paper, we seek to gain an indepth understanding and provide analytical proofs of $\mathrm{MKC}$ 's delayed stability in the control-theoretic sense. We start our investigation with its local properties in the next section.

\section{Delayed Local Stability}

\section{A. Delayed Linear Stability}

Before focusing on $\mathrm{MK}_{\mathrm{C}}$, we first show the existence of a class of delayed control systems, whose stability directly follows from that of the corresponding undelayed systems, and later show that $\mathrm{M}_{\mathrm{C}}$ falls into this category. Examine the following theorem that formalizes the generic law mentioned above.

Theorem 1: Assume an undelayed linear system $\mathcal{L}$ with $N$ flows:

$$
x_{i}(n)=\sum_{j=1}^{N} a_{i j} x_{j}(n-1) .
$$

If the coefficient matrix $A=\left(a_{i j}\right)$ is real-valued and symmetric, then system $\mathcal{L}_{D}$ with arbitrary directional delays:

$$
x_{i}(n)=\sum_{j=1}^{N} a_{i j} x_{j}\left(n-D_{j} \rightarrow-D_{i}^{\leftarrow}\right)
$$

is asymptotically stable if and only if $\mathcal{L}$ is stable.

Proof: See [16].

Theorem 1 opens an avenue for inferring stability of a delayed linear system based on the stability properties and coefficient matrix $A$ of the corresponding undelayed system. Moreover, Theorem 1 is also applicable to nonlinear systems as we show in the following corollary.

Corollary 1: Assume an undelayed $N$-dimensional nonlinear system $\mathcal{N}$ :

$$
x_{i}(n)=f_{i}\left(x_{1}(n-1), x_{2}(n-1), \cdots, x_{N}(n-1)\right),
$$

where $\left\{f_{i} \mid f_{i}: \mathbb{R}^{N} \rightarrow \mathbb{R}\right\}$ is the set of nonlinear functions defining the system. If the Jacobian matrix $J$ of this system is symmetric and real-valued, system $\mathcal{N}_{D}$ with arbitrary delay:

$$
\begin{array}{r}
x_{i}(n)=f_{i}\left(x_{1}\left(n-D_{1}^{\rightarrow}-D_{i}^{\leftarrow}\right), x_{2}\left(n-D_{2}^{\rightarrow}-D_{i}^{\leftarrow}\right)\right. \\
\left.\cdots, x_{N}\left(n-D_{N}^{\rightarrow}-D_{i}^{\leftarrow}\right)\right)
\end{array}
$$

is locally asymptotically stable in the stationary point $\mathrm{x}^{*}$ if and only if $\mathcal{N}$ is stable in $\mathrm{x}^{*}$.

Based on the principle demonstrated above, we next examine local stability of $\mathrm{MKC}$ under random (heterogeneous) feedback delays.

\section{B. Local Asymptotic Stability of $M K_{C}$}

We first derive the condition of local asymptotic stability of $\mathrm{MKC}(4)-(6)$, whose feedback generating function $p(n)$ is assumeed to be differentiable in the stationary point and has the same first-order partial derivative for all end-users. Following that, we will specialize this result to $\mathrm{EMKC}_{\mathrm{C}}$ with the particular packet loss function (7).

We approach this problem by applying Theorem 1, whose first step is to show stability of the following undelayed system:

$$
x_{i}(n)=(1-\beta p(n-1)) x_{i}(n-1)+\alpha,
$$

where $p(n)$ is the undelayed version of (6).

Theorem 2: The undelayed $N$-dimensional $\mathrm{MKC}_{\mathrm{C}}$ system is locally asymptotically stable if and only if:

$$
\left\{\begin{array}{l}
0<\beta p^{*}<2 \\
0<\beta p^{*}+\left.\beta N x^{*} \frac{\partial p}{\partial x_{i}}\right|_{\mathbf{x}^{*}}<2
\end{array},\right.
$$

where $x^{*}$ is the fixed point of each individual user, vector $\mathrm{x}^{*}=\left\langle x^{*}, x^{*}, \cdots, x^{*}\right\rangle$ is the fixed point of the entire system, and $p^{*}$ is the stationary packet loss.

Proof: See [16].

According to the proof of Theorem 2, Jacobian $J$ of the undelayed system (12) evaluated in the stationary point $\mathrm{x}^{*}$ is real-valued and symmetric. Thus, combining this observation with the result of Corollary 1, we obtain that heterogeneously delayed $\mathrm{MKC}$ is also locally asymptotically stable in $\mathrm{x}^{*}$.

Corollary 2: The heterogeneously delayed $\mathrm{MK}_{\mathrm{C}}$ system (4)-(6) is locally asymptotically stable if and only if (13) is satisfied.

Corollary 2 is a generic result that is applicable to $\mathrm{MKC}$ with a wide class of congestion-indicator functions $p(n)$. Note further that, for a given controller with pricing function $p(n)$, condition (13) is easy to verify and does not depend on feedback delays. This is in contrast to all current studies [2], [10], [13], [14], whose results are dependent on individual feedback delay $D_{i}$. We next extend the above analysis to $\mathrm{EMKC}_{\mathrm{C}}$ with the particular feedback given in (7).

Theorem 3: The heterogeneously delayed $\mathrm{E}_{\mathrm{M}} \mathrm{K}_{\mathrm{C}}$ system defined by (4) and (7) is locally asymptotically stable if and only if $0<\beta<2$. 


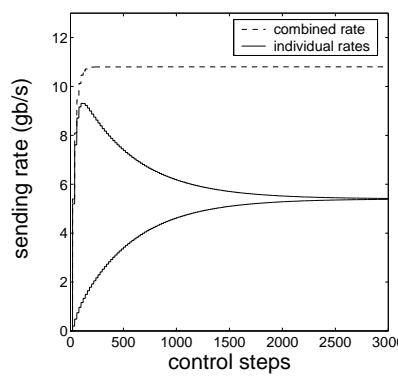

(a)

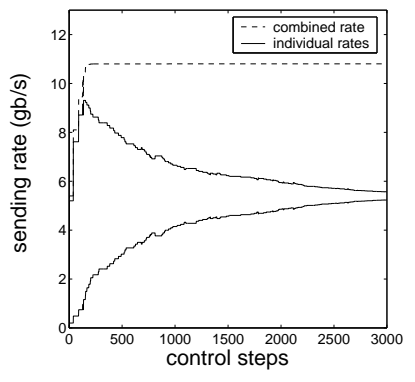

(b)
Fig. 2. The delayed behavior of MKC: (a) dynamics under constant delay $D=20$ time units; (b) dynamics under delays randomly distributed between 1 and 100 time units.

\section{Proof: See [16]}

To better understand the implication of this theorem, consider an illustration in Figure 2, where two $\mathrm{EMKC}_{\mathrm{K}}$ flows ( $\alpha=200 \mathrm{mb} / \mathrm{s}$ and $\beta=0.5$ ) share a bottleneck link of capacity $10 \mathrm{gb} / \mathrm{s}$. Recall that for the same setup, the classic Kelly control is unstable for any delay $D>3$ time units [16]. In the first example, the feedback delay is 20 time units for each flow, while in the second example, delays of each flow randomly fluctuate between 1 and 100 time units at each control step. As seen in both examples in Figure 2 , full link utilization is reached without oscillations (even though individual delays are different) and eventually the two flows share the resource fairly. These simulation results support our previous conclusion that $\mathrm{EMK}_{\mathrm{K}}$ is a stable and fair controller under random delays, which is a requirement for any practical method to be used in the current Internet.

Additional simulation results suggest that $\mathrm{EMKC}_{\mathrm{C}}$ is also globally asymptotically stable since flows starting from any initial conditions always converge to the same unique fixed point $x^{*}$. Thus, we are motivated to analytically prove this observation by conducting the global analysis of $\mathrm{EMKC}_{\mathrm{C}}$ in the next section.

\section{Global Stability under Constant Delay}

Recall that global asymptotic stability of a nonlinear dynamic system requires both Lyapunov stability and global quasi-asymptotic stability (whose definition follows later) in the unique stable fixed point [1]. Note that we proved local asymptotic stability of $\mathrm{EMKC}_{\mathrm{C}}$ in the preceding section, which implies Lyapunov stability of the system. Thus, our remaining task is to prove that $\mathrm{EMKC}_{\mathrm{C}}$ will converge to the unique fixed point regardless of its initial conditions. To accomplish this, we first consider several auxiliary results.

\section{A. Preliminaries}

We start with a very simple lemma.

Lemma 1: For an arbitrary sequence $v_{n}$ such that $v_{n} \rightarrow$ 0 for $n \rightarrow \infty$ and another sequence $\alpha_{n}$ such that $\forall n>$ $n_{0}:\left|\alpha_{n}\right|<1-\varepsilon$, where $\varepsilon>0$, the following recurrence converges to zero regardless of the value of $x_{0}$ :

$$
x_{n}=\alpha_{n} x_{n-1}+v_{n} .
$$

Proof: We define a new set of variables such that $y_{n}=x_{n+n_{0}}, \beta_{n}=\alpha_{n+n_{0}}$, and $u_{n}=v_{n+n_{0}}$ to shift system (14) by $n_{0}$ time units forward and skip the transient region of the evolution of $x_{n}$ when $\alpha_{n}$ can potentially be larger than 1 :

$$
y_{n}=\beta_{n} y_{n-1}+u_{n}
$$

Using these assignments, $\left|\beta_{n}\right|$ is less than $1-\varepsilon$ for all $n \geq 0$. We next demonstrate that sequence $y_{n}$ converges to zero, which implies that $x_{n}$ does too. Recursively expanding $y_{n}$, for $n \geq 2$, we get:

$$
y_{n}=\prod_{i=1}^{n} \beta_{i} y_{0}+u_{n}+\sum_{i=1}^{n-1} u_{i} \prod_{j=i+1}^{n} \beta_{j} .
$$

For convenience of presentation, let

$$
\begin{aligned}
& S_{1}(n)=\prod_{i=1}^{n} \beta_{i} y_{0}+u_{n}, \\
& S_{2}(n)=\sum_{i=1}^{n-1}\left(u_{i} \prod_{j=i+1}^{n} \beta_{j}\right) .
\end{aligned}
$$

Since $\left|\beta_{n}\right|<1-\varepsilon$ and $u_{n}$ is a time-shifted version of $v_{n}$, we immediately obtain that $S_{1}(n) \rightarrow 0$ as $n \rightarrow \infty$. We next examine $S_{2}(n)$ and show that it also tends to zero for large $n$. Re-writing (18):

$$
\left|S_{2}(n)\right| \leq \sum_{i=1}^{n-1}\left(\left|u_{i}\right| \prod_{j=i+1}^{n}\left|\beta_{j}\right|\right) .
$$

Again since $\left|\beta_{n}\right|<1-\varepsilon$, we have:

$$
\left|S_{2}(n)\right| \leq \sum_{i=1}^{n-1}\left|u_{i}\right|(1-\varepsilon)^{n-i}=G_{1}(n)+G_{2}(n),
$$

where we define:

$$
\begin{aligned}
& G_{1}(n)=\sum_{i=1}^{n / 2}\left|u_{i}\right|(1-\varepsilon)^{n-i}, \\
& G_{2}(n)=\sum_{i=n / 2+1}^{n-1}\left|u_{i}\right|(1-\varepsilon)^{n-i} .
\end{aligned}
$$

To show that both $G_{1}(n)$ and $G_{2}(n)$ converge to zero, we need the following notations:

$$
\begin{aligned}
& m_{1}(n)=\max \left(\left|u_{1}\right|, \ldots,\left|u_{n / 2}\right|\right) \\
& m_{2}(n)=\max \left(\left|u_{n / 2+1}\right|, \ldots,\left|u_{n-1}\right|\right) .
\end{aligned}
$$


Then we have:

$$
\begin{aligned}
G_{1}(n) & \leq m_{1}(n) \sum_{i=1}^{n / 2}(1-\varepsilon)^{n-i} \\
& =m_{1}(n) \sum_{j=n / 2}^{n-1}(1-\varepsilon)^{j} \\
& =m_{1}(n)\left(\sum_{j=0}^{n-1}(1-\varepsilon)^{j}-\sum_{j=0}^{n / 2-1}(1-\varepsilon)^{j}\right) \\
& =m_{1}(n) \frac{(1-\varepsilon)^{n}-(1-\varepsilon)^{n / 2}}{\varepsilon} .
\end{aligned}
$$

Since $m_{1}(n)$ is bounded and $0<\varepsilon<1, G_{1}(n) \rightarrow 0$. For $G_{2}(n)$, we have:

$$
\begin{aligned}
G_{2}(n) & \leq m_{2}(n) \sum_{i=n / 2+1}^{n-1}(1-\varepsilon)^{n-i} \\
& \leq m_{2}(n) \sum_{i=0}^{\infty}(1-\varepsilon)^{i}=\frac{m_{2}(n)}{\varepsilon} .
\end{aligned}
$$

Notice that since both $u_{n / 2}$ and $u_{n}$ converge to zero, then so must $m_{2}(n)$. Therefore, we get $G_{2}(n) \rightarrow 0$, which leads to $S_{2}(n) \rightarrow 0$ and hence $y_{n} \rightarrow 0$.

We next present our main result of this section.

Theorem 4: Assume a nonlinear system

$$
x_{n}=f\left(x_{n-1}, y_{n-1}\right),
$$

where function $f(x, y)$ is linear in both arguments:

$$
f(x, y)=a+b x+c y+d x y
$$

for some constants $a-d$. Further assume that $y_{n}$ converges to a stationary point $y^{*}$ as $n \rightarrow \infty$ and form another system, which replaces $y_{n}$ with $y^{*}$ in (27):

$$
\tilde{x}_{n}=f\left(\tilde{x}_{n-1}, y^{*}\right) \text {. }
$$

Then, system (27) converges if and only if system (29) converges, in which case the two stationary points are the same regardless of the initial points $x_{0}$ and $\tilde{x}_{0}$ in which each system is started:

$$
\lim _{n \rightarrow \infty}\left|x_{n}-\tilde{x}_{n}\right|=0 .
$$

Proof: We again only prove the sufficient condition. The necessary condition follows by reversing the order of steps. First notice that system (29) is stable (bounded) if and only if $\left|b+d y^{*}\right|<1$. Next denote by $\Delta x_{n}$ the absolute distance between the trajectories of the two systems at time $n$ :

$$
\Delta x_{n}=x_{n}-\tilde{x}_{n} .
$$

Further let $\Delta y_{n}=y_{n}-y^{*}$ be the distance of $y_{n}$ from its stationary point. Then we can write:

$$
\begin{aligned}
\Delta x_{n+1} & =x_{n+1}-\tilde{x}_{n+1} \\
& =f\left(x_{n}, y_{n}\right)-f\left(\tilde{x}_{n}, y^{*}\right) \\
& =f\left(x_{n}, y_{n}\right)-f\left(\tilde{x}_{n}, y_{n}\right)+f\left(\tilde{x}_{n}, y_{n}\right)-f\left(\tilde{x}_{n}, y^{*}\right) \\
& =\left(b+d y_{n}\right) \Delta x_{n}+\left(c+d \tilde{x}_{n}\right) \Delta y_{n} .
\end{aligned}
$$

(32) properties of a dynamic system.
Next notice that (32) defines a recursive relationship on $\Delta x_{n}$ :

$$
\Delta x_{n}=\alpha_{n} \Delta x_{n-1}+v_{n}
$$

where $\alpha_{n}=b+d y_{n}$ and $v_{n}=\left(c+d \tilde{x}_{n}\right) \Delta y_{n}$. First, since $\tilde{x}_{n}$ is bounded and $\Delta y_{n} \rightarrow 0$ as $n \rightarrow \infty$, we have $v_{n} \rightarrow 0$ for large $n$. Second, since $\left|b+d y^{*}\right|<1$, there exists such $\varepsilon$ that:

$$
\left|b+d y^{*}\right|<1-2 \varepsilon .
$$

Since $y_{n} \rightarrow y^{*}$, there exists such $n_{0}$ that $\forall n>n_{0}$, sequence $\alpha_{n}$ is bounded by the following:

$$
\left|\alpha_{n}\right|=\left|b+d y_{n}\right|<1-\varepsilon, \forall n>n_{0} .
$$

Thus, system (33) satisfies the conditions of Lemma 1 and therefore converges to zero as $n \rightarrow \infty$.

\section{B. $E_{M} K_{C}$}

Our next two results, respectively, show global stability of the combined rate $X(n)$ and convergence of packet loss $p(n)$ to $p^{*}$ regardless of the behavior of flow rates $x_{i}(n)$.

Lemma 2: When $0<\beta<2$, the combined rate $X(n)$ of $\mathrm{EMK}_{\mathrm{C}}$ is globally asymptotically stable under constant delay and converges to $X^{*}=C+N \alpha / \beta$ at an exponential rate.

Proof: Assume that feedback delay $D$ is constant. Combining (4)-(7) and taking the summation for all $N$ flows, we get that EMKC's combined rate $X(n)=\sum_{i} x_{i}(n)$ forms a linear system:

$$
\begin{aligned}
X(n) & =\left(1-\beta \frac{X(n-D)-C}{X(n-D)}\right) X(n-D)+N \alpha \\
& =(1-\beta) X(n-D)+\beta C+N \alpha .
\end{aligned}
$$

It is clear that the above linear system is stable if and only if $0<\beta<2$. Since convergence of linear systems implies global asymptotic stability, we can conclude that $X(n)$ is globally stable regardless of individual flow trajectories $x_{i}(n)$.

We next show the convergence speed of $X(n)$. Recursively expanding the last equation, we have:

$$
X(n)=(1-\beta)^{\frac{n}{D}}\left(X_{0}-X^{*}\right)+X^{*},
$$

where $X_{0}$ is the combined initial rate and $X^{*}=C+N \alpha / \beta$ is the combined stationary rate of all flows. Notice that for $0<\beta<2$, the first term in (37) approaches zero exponentially fast and $X(n)$ indeed converges to $X^{*}$.

Using (7), it is not difficult to see that $p(n)$ can be expressed as $p(n)=1-C / X(n)$. Combining this observation with the result of Lemma 2, we immediately have the following corollary.

Corollary 3: When $0<\beta<2$, $\mathrm{E}_{\mathrm{M}} \mathrm{K}_{\mathrm{C}}$ 's packet loss $p(n)$ converges to $p^{*}=N \alpha /(C \beta+N \alpha)$ regardless of the initial rates of the flows or their individual rates $x_{i}(n)$.

Before showing global stability of $\mathrm{EMK}_{\mathrm{C}}$, we first review the following stability concept that describes asymptotic 
Definition 1: [1] A point $\mathrm{x}^{*}$ is globally quasiasymptotically stable if and only if for all $\varepsilon>0$ there exists $n_{0}$ such that for all $n>n_{0}:\left|\mathbf{x}(n)-\mathbf{x}^{*}\right|<\varepsilon$ regardless of the initial point $\mathbf{x}(0)$.

According to Corollary $1, \mathrm{EMKC}_{\mathrm{K}}$ is locally quasiasymptotically stable in its unique fixed point $\mathrm{x}^{*}$. In what follows, we prove that each individual flow rate $x_{i}(n)$ is globally quasi-asymptotically stable, which implies that the entire system of flows $\mathbf{x}(n)=\left\langle x_{1}(n), \ldots, x_{N}(n)\right\rangle$ also exhibits global quasi-asymptotic stability.

Theorem 5: Assuming an $N$-flow $\mathrm{EMKC}_{\mathrm{K}}$ system with constant delay $D$ and an arbitrary initial point $\mathbf{x}(0)=$ $\left\langle x_{1}(0), \ldots, x_{N}(0)\right\rangle$, each flow $x_{i}(n)$ converges to $x^{*}=$ $C / N+\alpha / \beta$ if and only if $0<\beta<2$.

Proof: We start with the sufficient condition. Under constant delay $D$, each $\mathrm{EMKC}_{\mathrm{C}}$ flow activates a rate adjustment every $D$ time units. Thus, we can define a new set of flows $\left\{u_{i}(t)\right\}$, which operate in time units scaled by a factor of $D$. Under this notation, we can write $x_{i}(n)=$ $u_{i}(n / D)=u_{i}(t)$ and $x_{i}(n-D)=u_{i}(n / D-1)=u_{i}(t-1)$. Notice that $u_{i}(t)$ has the same exact stability properties as $x_{i}(n)$. Select an arbitrary flow $u_{i}$ and focus on its stability:

$$
u_{i}(t)=f\left(u_{i}(t-1), p(t-1)\right),
$$

where $p(t)$ is the packet loss at time $t$ and $f(x, y)$ is given by:

$$
f(x, y)=(1-\beta y) x+\alpha .
$$

Then form a new system:

$$
\tilde{u}(t)=f\left(\tilde{u}(t-1), p^{*}\right)=\left(1-\beta p^{*}\right) \tilde{u}(t-1)+\alpha,
$$

where $\tilde{u}(0)=u_{i}(0)$, and notice that the solution to this recurrence is stable if and only if $\left|b+d y^{*}\right|=\left|1-\beta p^{*}\right|<1$. This condition is automatically satisfied using the proof of EMKC's local stability in Theorem 3. According to Corollary 3 , we notice that $p(t)$ converges to its unique stationary point $p^{*}$ regardless of $\mathbf{x}(0)$. Since (39) is linear in each argument, we can apply Theorem 4 and immediately obtain that $u_{i}(n) \rightarrow \tilde{u}^{*}=C / N+\alpha / \beta$ and is therefore quasiasymptotically stable regardless of the initial points $u_{i}(0)$ or $\mathbf{x}(0)$. Repeating the same argument for all flows $i$, we establish their individual convergence.

The necessity of condition $0<\beta<2$ directly follows from local properties of $\mathrm{EMKC}_{\mathrm{C}}$ in Corollary 3.

Combining $\mathrm{EMK}_{\mathrm{C}}$ 's Lyapunov stability and its global quasi-asymptotic stability, we have the following result.

Corollary 4: $\mathrm{EM}_{\mathrm{C}}$ is globally asymptotically stable under constant feedback delay $D$ if and only if $0<\beta<2$.

\section{CONCLUSION}

This paper offered a comprehensive stability analysis of a new congestion controller called $\mathrm{MKC}_{\mathrm{C}}$, which is proven to be locally asymptotically stable with arbitrary (heterogeneous) feedback delays under easily verifiable conditions. This property makes $\mathrm{MKC}$ a highly appealing platform for congestion control in future high-speed networks with heterogeneous users. Moreover, we proposed a negative packet-loss feedback function to be used in conjunction with $\mathrm{MKC}_{\mathrm{C}}$ and called the resulting controller $\mathrm{EMKC}_{\mathrm{C}}$. We proved that $\mathrm{EMK}_{\mathrm{C}}$ achieves both RTT-independent stability and fairness and converges to link utilization exponentially fast.

Our investigation of global stability shows that all $\mathrm{EMKC}_{\mathrm{K}}$ flows converge to their unique stationary points regardless of the initial point in which the system is started. We proved this fact for constant delays $D$ and our future work is to extend the analysis to heterogenous delays.

\section{REFERENCES}

[1] P. Glendinning. Stability, Instability and Chaos: an Introduction to the Theorey of Nonlinear Differential Equations. Cambridge University Press, 1994.

[2] R. Johari and D. K. H. Tan, "End-to-End Congestion Control for the Internet: Delays and Stability," IEEE/ACM Transactions on Networking, 9(6):818-832, December 2001.

[3] K. Kar, S. Sarkar, and L. Tassiulas, "A Simple Rate Control Algorithm for Maximizing Total User Utility," IEEE INFOCOM, April 2001.

[4] F. P. Kelly, A. K. Maulloo, and D. K. H. Tan, "Rate Control for Communication Networks: Shadow Prices, Proportional Fairness and Stability," Journal of the Operational Research Society, 49(3):237-252, March 1998.

[5] S. Kunniyur and R. Srikant, "Analysis and Design of an Adaptive Virtual Queue (AVQ) Algorithm for Active Queue Management," ACM SIGCOMM, August 2001.

[6] S. Kunniyur and R. Srikant, "End-to-End Congestion Control Schemes: Utility Functions, Random Losses and ECN Marks," IEEE/ACM Transactions on Networking, 11(5):689702, October 2003.

[7] S. Kunniyur and R. Srikant, "Stable, Scalable, Fair Congestion Control and AQM Schemes that Achieve High Utilization in the Internet," IEEE Transactions on Automatic Control, 48(11):2024-2029, November 2003.

[8] S. H. Low, "A Duality Model of TCP and Queue Management Algorithms," IEEE/ACM Transactions on Networking, 11(4):525-536, August 2003.

[9] S. H. Low and D. E. Lapsley, "Optimization Flow Control I: Basic Algorithm and Convergence," IEEE/ACM Transactions on Networking, 7(6):861-874, December 1999.

[10] L. Massoulié, "Stability of Distributed Congestion Control with Heterogeneous Feedback Delays," IEEE/ACM Transactions on Networking, 47(6):895-902, June 2002.

[11] L. Massoulié and J. Roberts, "Bandwidth Sharing: Objectives and Algorithms," IEEE INFOCOM, March 1999.

[12] S. Shakkottai and R. Srikant, "Mean FDE Models for Internet Congestion Control Under a Many-Flows Regime," Technical report, Coordinated Science Laboratory, University of Illinois at Urbana-Champaign, Augest 2001.

[13] G. Vinnicombe, "On the Stability of End-to-End Congestion Control for the Internet," Technical Report CUED/FINFENG/TR.398, University of Cambridge, December 2000.

[14] G. Vinnicombe, "Robust congestion control for the Internet," Technical report, University of Cambridge, 2002.

[15] L. Ying, G. E. Dullerud, and R. Srikant, "Global Stability of Internet Congestion Control with Heterogeneous Delays," American Control Conference, June 2004.

[16] Y. Zhang, S.-R. Kang, and D. Loguinov, "Delayed Stability and Performance of Distributed Congestion Control," ACM SIGCOMM, August 2004. 